\title{
CrystEngComm
}

Cite this: CrystEngComm, 2014, 16, 7043

Received 20th February 2014 Accepted 20th May 2014

DOI: $10.1039 / c 4 c e 00375 f$

www.rsc.org/crystengcomm

\section{Tuning the structure of metal phosphonates using uncoordinating methyl group: syntheses, structures and properties of a series of metal diphosphonates $\dagger$}

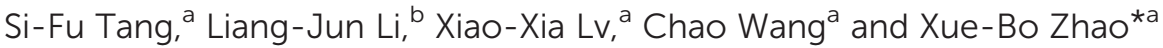

\begin{abstract}
Eight new transition metal diphosphonates, namely, $\left[\mathrm{Mn}\left(\mathrm{H}_{2} \mathrm{~L}\right)\left(\mathrm{H}_{2} \mathrm{O}\right)_{2}\right]\left[\left(\mathrm{H}_{2} \mathrm{O}\right)_{2}\right](1)$, $\left[\mathrm{Co}\left(\mathrm{H}_{2} \mathrm{O}\right)_{6}\right]\left[\mathrm{H}_{2} \mathrm{~L}\right]\left[\left(\mathrm{H}_{2} \mathrm{O}\right)_{2}\right]$ (2), [Co( $\left.\left.\mathrm{H}_{2} \mathrm{O}\right)_{6}\right]\left[\left(\mathrm{H}_{3} \mathrm{~L}\right)_{2}\right]\left[\left(\mathrm{H}_{2} \mathrm{O}\right)_{2}\right](3),\left[\mathrm{Ni}\left(\mathrm{H}_{2} \mathrm{O}\right)_{6}\right]\left[\mathrm{H}_{2} \mathrm{~L}\right]\left[\left(\mathrm{H}_{2} \mathrm{O}\right)_{2}\right](4),\left[\mathrm{Cu}\left(\mathrm{H}_{2} \mathrm{~L}\right)\right]\left[\left(\mathrm{H}_{2} \mathrm{O}\right)_{2}\right](5),\left[\mathrm{Zn}\left(\mathrm{H}_{2} \mathrm{O}\right)_{6}\right]\left[\left(\mathrm{H}_{3} \mathrm{~L}\right)_{2}\right]$ $\left[\left(\mathrm{H}_{2} \mathrm{O}\right)_{2}\right](6),\left[\mathrm{Zn}\left(\mathrm{H}_{2} \mathrm{~L}\right)\left(\mathrm{H}_{2} \mathrm{O}\right)_{2}\right](7)$ and $\left[\mathrm{Cd}\left(\mathrm{H}_{2} \mathrm{~L}\right)\left(\mathrm{H}_{2} \mathrm{O}\right)_{2}\right](8)$, have been synthesized hydrothermally from 2,5-dimethyl-1,4-phenylenediphosphonic acid $\left(\mathrm{H}_{4} \mathrm{~L}\right)$ and thoroughly characterized using EA, IR, TGA, powder and single-crystal XRD, luminescence and magnetism methods. The single-crystal X-ray diffraction measurements indicate that the diphosphonate ligands adopt three kinds of coordination modes which have a huge influence on the structure formation. Compounds 1 and 8 are isostructural and feature 3D framework structures which are constructed by the connectivity of $M_{6}(M=M n$ and $C d)$ octahedrons and tetradentate organic linkers. Compounds 2 and 4, 3 and 6 are also isostructural, but have isolated mononuclear structures. Compound 5 exhibits 2D layered structure, in which fourconnected copper(II) centers are bridged by tetradentate diphosphonate ligands. Compound 7 has a 1D infinite chain structure which is constructed from $\mathrm{ZnO}_{4}$ tetrahedrons and bidentate bridging diphosphonate ligands. It is also found that compounds 6-8 display interesting luminescent properties, whereas 1 shows an antiferromagnetic property.
\end{abstract}

\section{Introduction}

Metal phosphonates have attracted a great deal of attention due to their interesting structures and potential applications in the field of optics, catalysis, magnetism, gas storage/ separation, and so on. ${ }^{1}$ Compared with the carboxylic group, the phosphonic group $-\mathrm{PO}_{3} \mathrm{H}_{2}$ has an additional coordinating site which allows it to bind to metal centers with more coordination modes. In the field of carboxylate-based MOFs, isoreticular synthesis has been widely used to alter the chemical composition, functionality, and molecular dimensions systematically without changing the underlying topology. ${ }^{2}$ However, in the field of metal phosphonates, isoreticular synthesis is rarely observed ${ }^{3}$ due to its variable coordination modes which largely depend on the $\mathrm{pH}$ value, temperature,

\footnotetext{
${ }^{a}$ Qingdao Institute of Bioenergy and Bioprocess Technology, Chinese Academy of Sciences, Qingdao 266101, China. E-mail: zhaoxb@qibebt.ac.cn; Fax: 860532 80662728; Tel: 86053280662729

${ }^{b}$ Institute of Unconventional Oil \& Gas and Novel Energy, China University of Petroleum (East China), Shandong 266580, China

$\dagger$ Electronic supplementary information (ESI) available: $\mathrm{O}-\mathrm{M}-\mathrm{O}$ angles, $\mathrm{O}-\mathrm{H} \cdots \mathrm{O}$ bonds, PXRD and X-ray crystallographic files for 1-8 in CIF format. CCDC 986873-986879 and 996815. For ESI and crystallographic data in CIF or other electronic format see DOI: 10.1039/c4ce00375f
}

template, metal/ligand molar ratio, and so on. ${ }^{4}$ As one type of inorganic-organic hybrid material, it is important to predict the structures and understand the structure-property relationship of metal phosphonates so that materials with specific structures and properties can be designed and prepared. To fabricate new metal phosphonates with novel structures and properties, auxiliary second ligands such as 2,2'-bipy, 4,4'-bipy, 1,10-phen and oxalic acid have usually been added and proved to be an effective strategy. ${ }^{5}$ Another effective strategy is the decoration of the phosphonate ligand with functional coordinating groups such as hydroxyl, pyridine, carboxylic, sulfonyl and crown ether groups, which can provide additional coordination sites and lead to a large variety of new assemblies. ${ }^{6}$ Uncoordinating group, such as methyl group, doesn't participate in coordination but can tune the solubility, crystallinity and conformation of the phosphonate ligand and lead to new species. In our recent papers, we found that the attachment of three methyl groups to one flexible trisphosphonate ligand, benzene-1,3,5-triyltris (methylene)triphosphonic acid, had a major effect on the structure formation of one metal phosphonate, ${ }^{7,8}$ suggesting an effective strategy to tune the structure and property of metal phosphonates. It is interesting to test whether this kind of phenomenon happens in a rigid phosphonate 
system. 1,4-Phenylenediphosphonic acid has long been employed as a ligand for the construction of metal phosphonates with $1 \mathrm{D}, 2 \mathrm{D}$, and 3D structures bearing interesting magnetic and luminescent properties. ${ }^{9,10}$ The attachment of two methyl groups to the central benzene ring leads to the formation one new diphosphonate ligand, 2,5-dimethyl-1,4-phenylenediphosphonic acid $\left(\mathrm{H}_{4} \mathrm{~L}\right)$. It was reacted with transition metal salts and resulted in eight new metal diphosphonates, namely, $\left[\mathrm{Mn}\left(\mathrm{H}_{2} \mathrm{~L}\right)\left(\mathrm{H}_{2} \mathrm{O}\right)_{2}\right]\left[\left(\mathrm{H}_{2} \mathrm{O}\right)_{2}\right]$ (1), $\left[\mathrm{Co}\left(\mathrm{H}_{2} \mathrm{O}\right)_{6}\right]\left[\mathrm{H}_{2} \mathrm{~L}\right]\left[\left(\mathrm{H}_{2} \mathrm{O}\right)_{2}\right](2),\left[\mathrm{Co}\left(\mathrm{H}_{2} \mathrm{O}\right)_{6}\right]\left[\left(\mathrm{H}_{3} \mathrm{~L}\right)_{2}\right]\left[\left(\mathrm{H}_{2} \mathrm{O}\right)_{2}\right](3)$, $\left[\mathrm{Ni}\left(\mathrm{H}_{2} \mathrm{O}\right)_{6}\right]\left[\mathrm{H}_{2} \mathrm{~L}\right]\left[\left(\mathrm{H}_{2} \mathrm{O}\right)_{2}\right](4),\left[\mathrm{Cu}\left(\mathrm{H}_{2} \mathrm{~L}\right)\right]\left[\left(\mathrm{H}_{2} \mathrm{O}\right)_{2}\right](5),\left[\mathrm{Zn}\left(\mathrm{H}_{2} \mathrm{O}\right)_{6}\right]$ $\left[\left(\mathrm{H}_{3} \mathrm{~L}\right)_{2}\right]\left[\left(\mathrm{H}_{2} \mathrm{O}\right)_{2}\right](6),\left[\mathrm{Zn}\left(\mathrm{H}_{2} \mathrm{~L}\right)\left(\mathrm{H}_{2} \mathrm{O}\right)_{2}\right](7)$ and $\left[\mathrm{Cd}\left(\mathrm{H}_{2} \mathrm{~L}\right)\left(\mathrm{H}_{2} \mathrm{O}\right)_{2}\right]$ (8). Here, their syntheses, structures and properties are reported and discussed systematically.

\section{Experimental}

\section{Materials and instruments}

Triethylphosphite (Aldrich) and 1,3,5-trimethylbenzene (Aldrich) were dried by heating to reflux over sodium and distillation under an argon atmosphere in a two-necked flask equipped with a condenser and a solvent collector. Elemental analyses were performed on a Vario EL III elemental analyzer. IR spectra were recorded on a Nicolet 6700 FTIR spectrometer as $\mathrm{KBr}$ pellets in the range of $4000-400 \mathrm{~cm}^{-1}$. Powder X-ray patterns were obtained on a Bruker D8 Advance diffractometer using CuK $\alpha$ radiation. Solution ${ }^{1} \mathrm{H}$ NMR spectra were recorded on a Bruker AVANCE-III NMR (600 $\mathrm{MHz})$. Thermogravimetric analyses (TGA) were carried out on a NETZSCH STA $449 \mathrm{C}$ unit at a heating rate of $10^{\circ} \mathrm{C} \mathrm{min}^{-1}$ under a nitrogen atmosphere. Fluorescent analyses of compounds 6-8 were performed on a Fluoromax-4 spectrofluorometer. A variable temperature magnetic susceptibility measurement of compound $\mathbf{1}$ was obtained in the solid state using a Quantum Design SQUID MPMS-7 magnetometer operating at 1000 Oe.

\section{Single-crystal structure determination}

Single crystal X-ray diffraction measurements of compounds 1 to 8 were carried out on a Bruker SMART APEX II CCD diffractometer (MoK $\alpha$ radiation, $\lambda=0.71073 \AA$ ) at room temperature. SAINT was used for the integration of the intensity of the reflections and scaling. ${ }^{11}$ Absorption corrections were carried out with the program SADABS. ${ }^{12}$ Compound 4 grew as twin crystals so the TWINABS ${ }^{13}$ program was used to carry out absorption corrections and to produce the final HKLF 5 $($ BASF $=0.32722)$ format file for the refinement. The crystal structures were solved by direct methods using SHELXS. ${ }^{14}$ Subsequent difference Fourier analyses and least squares refinement with SHELXL- $97^{15}$ allowed for the location of the atom positions. In the final step of the crystal structure refinement, hydrogen atoms of idealized $-\mathrm{CH}_{2}$ and $-\mathrm{CH}_{3}$ groups were added and treated with the riding atom mode, and their isotropic displacement factors were chosen as $\mathbf{1 . 2}$ and 1.5 times the preceding carbon atom, respectively. One water molecule $(\mathrm{O} 2 \mathrm{w})$ in compound 1 is disordered over two positions and refined isotropically. $\mathrm{O} 4 \mathrm{w}$ in compounds 3 and 6 is split over two positions (0.5/0.5 occupancy) because of their higher displacement parameters. The hydrogen atoms on the water molecules of 1-8, except for $\mathrm{O} 2 \mathrm{~W}$ and $\mathrm{O}_{2} \mathrm{~W}^{\prime}$ in $\mathbf{1}$, $\mathrm{O} 4 \mathrm{w}$ and $\mathrm{O} 4 \mathrm{w}^{\prime}$ in 3 and 6 , were located from the difference Fourier map. The crystallographic details for compounds 1-8 are summarized in Table 1 . The data have been deposited in the Cambridge Crystallographic Data Centre (CCDC), deposition numbers CCDC 986873-986878, 996815, 986879 for compounds 1-8.

Synthesis of 2,5-dimethyl-1,4-phenylenediphosphonic acid $\left(\mathbf{H}_{4} \mathbf{L}\right)$. The diphosphonate ligand was synthesized by reacting 1,4-dibromo-2,5-dimethylbenzene (Energy Chemicals) with triethylphosphite using anhydrous $\mathrm{NiBr}_{2}$ (Alfa Aesar) as catalyst and followed by refluxing the obtained oil with conc. hydrochloric acid through an Arbuzov reaction. ${ }^{16}$ A powder product with a satisfying yield and purity can be isolated after filtration and washing with small portions of cold ethanol. ${ }^{1} \mathrm{HNMR}\left(\mathrm{DMSO}-\mathrm{d}^{6}\right.$ ) data for $\mathrm{H}_{4} \mathrm{~L}: \delta 7.6062-7.5743(\mathrm{Ph}-\mathrm{H}$, $\mathrm{m}, 2 \mathrm{H}) ; 2.4956\left(-\mathrm{CH}_{3}, \mathrm{~s}, 6 \mathrm{H}\right) . \mathrm{IR}\left(\mathrm{KBr}, \mathrm{cm}^{-1}\right): 3422.2(\mathrm{w}, \mathrm{b})$, 2768.2 (s, b), 2290.1 (m, b), 1636.6 (w), 1480.8 (w), 1457.0 (w), 1359.4 (m), 1219.5 (s), 1140.3 (vs), 1101.1 (s), 1031.5 (vs), 942.2 (vs), 904.6 (m), 818.4 (w), 767.7 (w), $624.6(\mathrm{~m}), 591.0(\mathrm{~s})$, $571.5(\mathrm{~m}), 490.9(\mathrm{~s}), 464.3(\mathrm{~m})$.

Synthesis of compound $\left[\mathrm{Mn}\left(\mathrm{H}_{2} \mathrm{~L}\right)\left(\mathrm{H}_{2} \mathrm{O}\right)_{2}\right]\left[\left(\mathrm{H}_{2} \mathrm{O}\right)_{2}\right](1) . \mathrm{H}_{4} \mathrm{~L}$ $(0.0887 \mathrm{~g}, 0.3333 \mathrm{mmol}), \mathrm{MnSO}_{4} \cdot \mathrm{H}_{2} \mathrm{O}(0.1127 \mathrm{~g}, 0.6666 \mathrm{mmol})$, and $6 \mathrm{~mL}$ of water were mixed and stirred in a Teflon-lined autoclave. Afterward, it was sealed and heated at $160{ }^{\circ} \mathrm{C}$ for 3 days and allowed to cool to room temperature over a time period of 24 hours. Pale pink block crystals ( $0.086 \mathrm{~g})$ were collected in a satisfying yield (38\%, based on the metal source) and washed with deionized water. Elemental analysis (\%) calcd for $\mathrm{C}_{8} \mathrm{H}_{18} \mathrm{MnO}_{10} \mathrm{P}_{2}$ (391.10): C 24.57, $\mathrm{H}$ 4.64; found: $\mathrm{C}$ 24.53, H 4.70. IR ( $\left.\mathrm{KBr}, \mathrm{cm}^{-1}\right): 3528.3$ (s, sh), 3385.1 (s, b), 2929.5 (s, sh), 2459.8 (m, b), 1640.3 (m), 1480.3 (m), 1387.1 (w), 1354.1 (m), 1260.9 (s), 1193.7 (s), 1133.0 (vs), 1034.6 (vs), 895.6 (vs), 739.5 (w), 625.5 (m), $591.4(\mathrm{~s}), 495.0(\mathrm{~m})$.

Synthesis of compound $\left[\mathrm{Co}\left(\mathrm{H}_{2} \mathrm{O}\right)_{6}\right]\left[\mathrm{H}_{2} \mathrm{~L}\right]\left[\left(\mathrm{H}_{2} \mathrm{O}\right)_{2}\right]$ (2). Equal moles of $\mathrm{H}_{4} \mathrm{~L}(0.0665 \mathrm{~g}), \mathrm{Co}(\mathrm{ac})_{2} \cdot 4 \mathrm{H}_{2} \mathrm{O}(0.0623 \mathrm{~g})$, and $3 \mathrm{~mL}$ of water were mixed and stirred in a Teflon-lined autoclave. Afterward, it was sealed and heated at $160{ }^{\circ} \mathrm{C}$ for 3 days and cooled to room temperature over a time period of 24 hours. Pink prism crystals were collected in a satisfying yield (64\%, based on the metal source) and washed with deionized water. Elemental analysis (\%) calcd for $\mathrm{C}_{8} \mathrm{H}_{26} \mathrm{CoO}_{14} \mathrm{P}_{2}$ (467.16): C 20.57, $\mathrm{H}$ 5.61; found: C 20.62, H 5.72. IR ( $\left.\mathrm{KBr}, \mathrm{cm}^{-1}\right): 3528.3$ (s, b), 3281.4 (vs, b), 2968.0 (m), 2933.6 (m), $2848.5(\mathrm{~m}), 2366.7(\mathrm{w})$, $1674.5(\mathrm{~m}), 1624.2(\mathrm{w}), 1476.4(\mathrm{~m}), 1456.9(\mathrm{w}), 1353.6(\mathrm{~m})$, $1227.9(\mathrm{w}), 1193.0(\mathrm{~m}), 1162.2$ (vs), $1035.9(\mathrm{~m}), 951.9$ (vs), $893.7(\mathrm{~s}), 707.5(\mathrm{w}), 618.2(\mathrm{~s}), 478.2(\mathrm{~m}), 456.5(\mathrm{w})$.

Synthesis of compound $\left[\mathrm{Co}\left(\mathrm{H}_{2} \mathrm{O}\right)_{6}\right]\left[\left(\mathrm{H}_{3} \mathrm{~L}\right)_{2}\right]\left[\left(\mathrm{H}_{2} \mathrm{O}\right)_{2}\right]$ (3). Compound 3 was synthesized using a method similar to that of 2 except for the replacement of $\mathrm{Co}(\mathrm{ac})_{2} \cdot 4 \mathrm{H}_{2} \mathrm{O}$ with $\mathrm{CoCl}_{2} \cdot 6 \mathrm{H}_{2} \mathrm{O}$. Elemental analysis (\%) calcd for $\mathrm{C}_{16} \mathrm{H}_{38} \mathrm{ZnO}_{20} \mathrm{P}_{4}$ (739.71): C 25.98, H 5.18; found: C 26.08, H 5.26. IR ( $\mathrm{KBr}$, $\left.\mathrm{cm}^{-1}\right)$ : $3405.1(\mathrm{~s}, \mathrm{~b}), 2967.3(\mathrm{~m}), 2934.8(\mathrm{~m}), 2386.2(\mathrm{w})$, 
Table 1 Crystallographic parameters of compounds 1-8

\begin{tabular}{|c|c|c|c|c|c|c|c|c|}
\hline Compound & 1 & 2 & 3 & 4 & 5 & 6 & 7 & 8 \\
\hline Formula & $\mathrm{C}_{8} \mathrm{H}_{18} \mathrm{MnO}_{10} \mathrm{P}_{2}$ & $\mathrm{C}_{8} \mathrm{H}_{26} \mathrm{CoO}_{14} \mathrm{P}_{2}$ & $\mathrm{C}_{16} \mathrm{H}_{38} \mathrm{CoO}_{20} \mathrm{P}_{4}$ & $\mathrm{C}_{8} \mathrm{H}_{26} \mathrm{NiO}_{14} \mathrm{P}_{2}$ & $\mathrm{C}_{8} \mathrm{H}_{14} \mathrm{CuO}_{8} \mathrm{P}_{2}$ & $\mathrm{C}_{16} \mathrm{H}_{38} \mathrm{ZnO}_{20} \mathrm{P}_{4}$ & $\mathrm{C}_{8} \mathrm{H}_{14} \mathrm{ZnO}_{8} \mathrm{P}_{2}$ & $\mathrm{C}_{8} \mathrm{H}_{14} \mathrm{CdO}_{8} \mathrm{P}_{2}$ \\
\hline Fw & 391.10 & 467.16 & 733.27 & 466.94 & 363.67 & 739.71 & 365.50 & 412.53 \\
\hline Space group & $C 2 / c$ & $P \overline{1}$ & $P 2(1) / c$ & $P \overline{1}$ & $P \overline{1}$ & $P 2(1) / c$ & Pnma & $C 2 / c$ \\
\hline$a(\AA)$ & $18.825(4)$ & $6.8159(18)$ & $12.363(2)$ & $6.7912(11)$ & $5.1671(10)$ & $12.3549(13)$ & $7.6143(12)$ & $19.781(4)$ \\
\hline$b(\AA)$ & $8.3912(16)$ & $9.734(3)$ & $7.8134(13)$ & $9.6878(16)$ & $6.3007(12)$ & $7.8244(8)$ & $22.461(4)$ & $6.7881(15)$ \\
\hline$c(\AA)$ & $9.2901(18)$ & $13.799(4)$ & $15.311(3)$ & $13.786(2)$ & $10.029(3)$ & $15.3271(16)$ & $7.7097(12)$ & $9.4449(19)$ \\
\hline$\alpha(\mathrm{deg})$ & 90 & $96.523(4)$ & 90 & $96.736(2)$ & $75.102(5)$ & 90 & 90 & 90 \\
\hline$\beta$ (deg) & $94.064(3)$ & $95.780(4)$ & $93.942(4)$ & $95.816(5)$ & $84.496(10)$ & $93.924(2)$ & 90 & $90.463(3)$ \\
\hline$\gamma(\mathrm{deg})$ & 90 & $91.060(4)$ & 90 & $91.082(5)$ & $76.610(3)$ & 90 & 90 & 90 \\
\hline$V\left(\AA^{3}\right)$ & $1463.8(5)$ & $904.6(4)$ & $1475.4(4)$ & $895.7(3)$ & $306.73(12)$ & $1478.2(3)$ & $1318.5(4)$ & $1268.2(5)$ \\
\hline$Z$ & 4 & 2 & 2 & 2 & 1 & 2 & 4 & 4 \\
\hline$D_{\text {calcd }} / \mathrm{g} \mathrm{cm}^{-3}$ & 1.775 & 1.715 & 1.651 & 1.731 & 1.969 & 1.662 & 1.841 & 2.161 \\
\hline Abs coeff $/ \mathrm{mm}^{-1}$ & 1.166 & 1.192 & 0.882 & 1.329 & 2.074 & 1.131 & 2.136 & 2.006 \\
\hline$F(000)$ & 804 & 486 & 762 & 488 & 185 & 768 & 744 & 816 \\
\hline Theta range & $2.659-24.998$ & $2.11-27.59$ & $2.667-26.428$ & $2.44-27.50$ & $2.103-24.998$ & $2.664-27.916$ & $1.81-27.65$ & $2.059-24.991$ \\
\hline Completeness/\% & 94.8 & 96.9 & 99.7 & 96.7 & 96.4 & 99.4 & 98.5 & 95.9 \\
\hline Reflns collected & 4587 & 8517 & 7876 & 4166 & 2400 & 8319 & 6620 & 3165 \\
\hline $\begin{array}{l}\text { Independent } \\
\text { reflns } / R_{\text {int }}\end{array}$ & $1250 / 0.0375$ & $4061 / 0.0501$ & $3020 / 0.0866$ & $4191 / 0.0000$ & $1063 / 0.0341$ & $3388 / 0.0499$ & $1544 / 0.0374$ & $1097 / 0.0458$ \\
\hline GOF on $F^{2}$ & 1.093 & 1.054 & 1.014 & 0.922 & 1.072 & 1.013 & 1.089 & 1.072 \\
\hline Final $R$ indices & 0.0355 & 0.0456 & 0.0592 & 0.0444 & 0.0379 & 0.0454 & 0.0322 & 0.0331 \\
\hline$[I>2 \sigma(I)]: R_{1}, \mathrm{w} R_{2}$ & 0.0941 & 0.1124 & 0.1266 & 0.0941 & 0.0937 & 0.1014 & 0.0925 & 0.0824 \\
\hline$R$ indices & 0.0385 & 0.0592 & 0.1145 & 0.0681 & 0.0471 & 0.0783 & 0.0367 & 0.0365 \\
\hline (all data): $R_{1}, \mathrm{w} R_{2}$ & 0.0963 & 0.1208 & 0.1552 & 0.1016 & 0.0998 & 0.1153 & 0.0953 & 0.0849 \\
\hline $\begin{array}{l}\text { Largest diff. peak } \\
\text { and hole } / \mathrm{e} \AA^{-3}\end{array}$ & $0.375 /-0.616$ & $0.534 /-0.540$ & $0.482 /-0.517$ & $0.534 /-0.627$ & $0.525 /-0.496$ & $0.380 /-0.515$ & $0.540 /-0.464$ & $0.615 /-0.515$ \\
\hline
\end{tabular}

$1625.6(\mathrm{~m}), 1476.7(\mathrm{~m}), 1353.6(\mathrm{~m}), 1261.4(\mathrm{~m}), 1193.0(\mathrm{~m})$, $1162.9(\mathrm{~s}), 1035.4(\mathrm{~m}), 951.2(\mathrm{~s}), 893.8(\mathrm{~m}), 708.1(\mathrm{~m})$, $618.2(\mathrm{~m}), 478.3(\mathrm{~m}), 456.4(\mathrm{w}), 439.4(\mathrm{w})$.

Synthesis of compound $\left[\mathrm{Ni}\left(\mathrm{H}_{2} \mathrm{O}\right)_{6}\right]\left[\mathrm{H}_{2} \mathrm{~L}\right]\left[\left(\mathrm{H}_{2} \mathrm{O}\right)_{2}\right](4) . \mathrm{H}_{4} \mathrm{~L}$ $(0.0665 \mathrm{~g}, 0.25 \mathrm{mmol}), \mathrm{NiSO}_{4} \cdot \mathrm{H}_{2} \mathrm{O}(0.1586 \mathrm{~g}, 0.25 \mathrm{mmol})$ and $10 \mathrm{~mL}$ of water were mixed in a Teflon-lined autoclave and stirred at room temperature for 30 minutes. The $\mathrm{pH}$ value was then altered to about 4 using $1 \mathrm{M} \mathrm{NaOH}$ and the autoclave was heated at $160{ }^{\circ} \mathrm{C}$ for 3 days. After slowly cooling to room temperature (24 hours) and washing with deionized water, green block crystals (0.049 g, yield: $42 \%$ ) were isolated. Elemental analysis (\%) calcd for $\mathrm{C}_{8} \mathrm{H}_{26} \mathrm{NiO}_{14} \mathrm{P}_{2}$ (466.94): C 20.58, H 5.61; found: C 20.63, H 5.69. IR ( $\left.\mathrm{KBr}, \mathrm{cm}^{-1}\right): 3452.2$ (vs, sh), 3382.6 (vs, sh), 3278.1 (vs, sh), 3171.3 (vs, b), 2444.4 (m, b), 1669.8 (m), 1479.1 (w), 1383.0 (vw), 1355.8 (w), 1259.3 (w), 1145.5 (vs), 1100.3 (m), 1025.6 (vs), 894.7 (s), 683.3 (m), 599.3 (s), $489.9(\mathrm{~m})$.

Synthesis of compound $\left[\mathrm{Cu}\left(\mathrm{H}_{2} \mathrm{~L}\right)\right]\left[\left(\mathrm{H}_{2} \mathrm{O}\right)_{2}\right]$ (5). Compound 5 was obtained using a method similar to that of 1 from a mixture of $\mathrm{H}_{4} \mathrm{~L}(0.0887 \mathrm{~g}, 0.3333 \mathrm{mmol}), \mathrm{CuSO}_{4} \cdot 5 \mathrm{H}_{2} \mathrm{O}(0.1665 \mathrm{~g}$, $0.6666 \mathrm{mmol}$ ), and $10 \mathrm{~mL}$ of $\mathrm{H}_{2} \mathrm{O}$. Blue-green plate crystals $(0.064 \mathrm{~g})$ were collected in a satisfying yield (53\%, based on $\mathrm{H}_{4} \mathrm{~L}$ ). Elemental analysis (\%) calcd for $\mathrm{C}_{8} \mathrm{H}_{14} \mathrm{CuO}_{8} \mathrm{P}_{2}$ (363.67): C 26.42, H 3.88; found: C 26.37, H 3.96. IR ( $\left.\mathrm{KBr}, \mathrm{cm}^{-1}\right): 3519.3$ (m, b), $3443.4(\mathrm{~m}, \mathrm{sh}), 3096.0(\mathrm{~m}, \mathrm{~b}), 1632.6(\mathrm{w}), 1482.4(\mathrm{vw})$, 1454.1 (w), $1374.9(\mathrm{w}), 1353.9(\mathrm{w}), 1208.1$ (m), $1154.2(\mathrm{~s})$, 1132.8 (vs), 1069.9 (vs), 915.8 (s), 897.0 (s), 702.7 (vw), 629.8 (w), $586.0(\mathrm{~s}), 527.7(\mathrm{~m}), 454.0(\mathrm{w})$.

Synthesis of compound $\left[\mathrm{Zn}\left(\mathrm{H}_{2} \mathrm{O}\right)_{6}\right]\left[\left(\mathrm{H}_{3} \mathrm{~L}\right)_{2}\right]\left[\left(\mathrm{H}_{2} \mathrm{O}\right)_{2}\right](6)$. The synthesis of 6 is similar to that of 2 except for the replacement of $\mathrm{Co}(\mathrm{ac})_{2} \cdot 4 \mathrm{H}_{2} \mathrm{O}$ with $\mathrm{ZnCl}_{2}$. Elemental analysis (\%) calcd for $\mathrm{C}_{16} \mathrm{H}_{38} \mathrm{ZnO}_{20} \mathrm{P}_{4}$ (739.74): C 25.98, $\mathrm{H} \mathrm{5.18;} \mathrm{found:}$ C 26.04, H 5.30. IR (KBr, cm ${ }^{-1}$ ): 3528.3 (s, b), 3281.4 (vs, b), $2968.0(\mathrm{~m}), 2933.6(\mathrm{~m}), 2848.5(\mathrm{~m}), 2366.7(\mathrm{w}), 1674.5(\mathrm{~m})$, $1624.2(\mathrm{w}), 1476.4(\mathrm{~m}), 1456.9(\mathrm{w}), 1353.6(\mathrm{~m}), 1227.9(\mathrm{w})$, 1193.0 (m), 1162.2 (vs), 1035.9 (m), 951.9 (vs), 893.7 (s), 707.5 (w), $618.2(\mathrm{~s}), 478.2(\mathrm{~m}), 456.5(\mathrm{w})$.

Synthesis of compound $\left[\mathrm{Zn}\left(\mathrm{H}_{2} \mathrm{~L}\right)\left(\mathrm{H}_{2} \mathrm{O}\right)_{2}\right]$ (7). A mixture of $\mathrm{H}_{4} \mathrm{~L}(0.1331 \mathrm{~g}, 0.5 \mathrm{mmol}), \mathrm{ZnCl}_{2}(0.1360 \mathrm{~g}, 1 \mathrm{mmol})$, and $10 \mathrm{~mL}$ of water were mixed and stirred in a Teflon-lined autoclave. Afterward, it was sealed and heated at $60^{\circ} \mathrm{C}$ for 7 days. Colorless small plate crystals were collected and washed with deionized water. Yield ( $77 \%$, based on the metal source). Elemental analysis (\%) calcd for $\mathrm{C}_{8} \mathrm{H}_{14} \mathrm{O}_{8} \mathrm{P}_{2} \mathrm{Zn}$ (365.52): C 26.29, H 3.86; found: C 26.36, H 3.93. IR ( $\left.\mathrm{KBr}, \mathrm{cm}^{-1}\right): 3331.1$ (vs, b), 2933.9 (s, sh), 2290.2 (m, b), 1788.8 (w), 1651.9 (m), 1476.9 (w), 1455.3 (w), 1388.5 (vw), 1353.9 (m), 1243.1 (m), 1140.0 (vs), $1085.0(\mathrm{~m}), 1035.0(\mathrm{~s}), 922.8(\mathrm{~s}), 895.3(\mathrm{~m}), 770.1(\mathrm{w}), 723.1(\mathrm{w})$, $626.0(\mathrm{~m}), 596.3(\mathrm{~m}), 467.8(\mathrm{w})$.

Synthesis of compound $\left[\mathrm{Cd}\left(\mathrm{H}_{2} \mathrm{~L}\right)\left(\mathrm{H}_{2} \mathrm{O}\right)_{2}\right]$ (8). A mixture of $\mathrm{H}_{4} \mathrm{~L}(0.0665 \mathrm{~g}, 0.25 \mathrm{mmol}), 3 \mathrm{CdSO}_{4} \cdot 8 \mathrm{H}_{2} \mathrm{O}(0.0641 \mathrm{~g}, 0.08 \mathrm{mmol})$, and $6 \mathrm{~mL}$ of water were mixed and stirred in a Teflon-lined autoclave. Afterward, it was sealed and heated at $160{ }^{\circ} \mathrm{C}$ for 3 days and allowed to cool to room temperature over a time period of 24 hours. Colorless block crystals $(0.079 \mathrm{~g}$, yield: $81 \%$, based on metal source) were collected and washed with deionized water. Elemental analysis (\%) calcd for $\mathrm{C}_{8} \mathrm{H}_{14} \mathrm{CdO}_{8} \mathrm{P}_{2}$ (412.53): C 23.29, $\mathrm{H}$ 3.42; found: $\mathrm{C}$ 23.19, H 3.50. IR (KBr, cm ${ }^{-1}$ ): 3386.1 (m, b), 2974.9 (m), 2927.5 (m), 2899.6 (m), 2413.6 (w, b), 2336.4 (w, b), 1561.6 (m), 1481.4 (w), 
1455.5 (w), 1385.7 (m), 1356.4 (m), 1184.4 (s), 1140.9 (s), 1046.2 (vs), 1018.7 (vs, sh), 875.5 (s), 758.7 (w), 599.4 (s), 477.9 (s), $438.3(\mathrm{w}), 424.6(\mathrm{w})$.

\section{Results and discussion}

\section{Structural description of compound 1}

Compound 1 crystallizes in the monoclinic space group $C 2 / c$ with four molecules in each unit cell. Each asymmetric unit contains one crystallographic independent Mn(II) ion lying on a twofold axis, half a diphosphonate ligand lying on an inversion centre, one aqua ligand and one lattice water molecule, indicating a formula of $\left[\mathrm{Mn}\left(\mathrm{H}_{2} \mathrm{~L}\right)\left(\mathrm{H}_{2} \mathrm{O}\right)_{2}\right]\left[\left(\mathrm{H}_{2} \mathrm{O}\right)_{2}\right]$. The $\mathrm{Mn}$ (II) ion is octahedrally coordinated by six symmetry related oxygen atoms including two water molecules and four phosphonate oxygen atoms from four diphosphonate ligands. The $\mathrm{Mn}-\mathrm{O}$ distances and $\mathrm{O}-\mathrm{Mn}-\mathrm{O}$ angles are observed in the range of 2.138(2)-2.249(2) $\AA$ and 87.12(9)-179.06(11) ${ }^{\circ}$, respectively (see Tables 2 and $\mathrm{S} 1, \mathrm{ESI} \dagger$ ). The $\mathrm{C}-\mathrm{P}$ bond length is found to be $1.806(3) \AA$, and the P-O lengths are in the range of 1.502(2)-1.580(2) $\AA$, which are all comparable to those of other reported metal phosphonates. Each of the phosphonate groups in the diphosphonate ligand is singly deprotonated and adopts a bidentate coordination mode bridging two Mn(II) ions (see mode a in Scheme 1 and Fig. 1). Therefore, the coordination mode of the diphosphonate ligand can be denoted as: $\mu^{4}: \eta^{0}: \eta^{1}: \eta^{1}: \eta^{0}: \eta^{1}: \eta^{1}$. Neighboring Mn(II) ions are bridged by phosphonate groups to form one-dimensional infinite chains along the $c$-direction (see Fig. 2a), which are cross-linked further by the organic linkers into a three-dimensional framework structure, leaving slits running along the $c$-direction (see Fig. $2 \mathrm{~b}$ and $\mathrm{S} 1, \mathrm{ESI} \dagger$ ). As expected, hydrogen interactions are observed between the aqua ligands and phosphonate groups (see Table S2, ESI $\dagger$ ).

\section{Structural description of compounds 2 and 4}

Two isostructural metal (Co(II) and Ni(II)) diphosphonates with a pillar layered structure have been synthesized from 1,4-phenylenediphosphonic acid. ${ }^{10 e}$ The reactions of 2,5-dimethyl-1,4-phenylenediphosphonic acid with $\mathrm{Co}(\mathrm{ac})_{2} \cdot 4 \mathrm{H}_{2} \mathrm{O}$ and $\mathrm{NiSO}_{4} \cdot \mathrm{H}_{2} \mathrm{O}$ lead to the formation of compounds 2 and 4 , which are also isostructural but are much different from the former reported ones. Here, only the structure of 2 will be discussed in detail. Different to compound 1, compound 2 crystallizes in the triclinic space group $P \overline{1}$ and the diphosphonate ligand is not involved in coordination with the metal ions. In the asymmetric unit there is one $\mathrm{Co}(\mathrm{II})$ ion, two half $\left(\mathrm{H}_{2} \mathrm{~L}\right)^{-}$ ligands lying about independent inversion centres, six coordinating aqua ligands and two lattice water molecules, corresponding to a formula of $\left[\mathrm{Co}\left(\mathrm{H}_{2} \mathrm{O}\right)_{6}\right]\left[\mathrm{H}_{2} \mathrm{~L}\right]$ $\left[\left(\mathrm{H}_{2} \mathrm{O}\right)_{2}\right]$ (see Fig. 3). The $\mathrm{Co}(\mathrm{II})$ center is six-coordinated by aqua ligands, forming an octahedral coordination environment. The Co-O distances are found to be in the range of 2.030(2)-2.138(2) $\AA$. The phosphonate group of the diphosphonate ligand is also singly-deprotonated (see
Table 2 Selected bond lengths of compounds $1-8^{a}$

1

\begin{tabular}{llll}
\hline $\mathrm{Mn}(1)-\mathrm{O}(2) \# 1$ & $2.138(2)$ & $\mathrm{Mn}(1)-\mathrm{O}(1) \# 3$ & $2.176(2)$ \\
$\mathrm{Mn}(1)-\mathrm{O}(2) \# 2$ & $2.38(2)$ & $\mathrm{Mn}(1)-\mathrm{O}(1 \mathrm{~W})$ & $2.249(2)$ \\
$\mathrm{Mn}(1)-\mathrm{O}(1)$ & $2.176(2)$ & $\mathrm{Mn}(1)-\mathrm{O}(1 \mathrm{~W}) \# 3$ & $2.249(2)$ \\
$\mathrm{O}(1)-\mathrm{P}(1)$ & $1.511(2)$ & $\mathrm{O}(3)-\mathrm{P}(1)$ & $1.581(2)$ \\
$\mathrm{O}(2)-\mathrm{P}(1)$ & $1.502(2)$ & $\mathrm{P}(1)-\mathrm{C}(3)$ & $1.805(3)$ \\
\hline 2 & & & \\
\hline $\mathrm{Co}(1)-\mathrm{O}(1 \mathrm{~W})$ & $2.030(2)$ & $\mathrm{Co}(1)-\mathrm{O}(4 \mathrm{~W})$ & $2.102(2)$ \\
$\mathrm{Co}(1)-\mathrm{O}(2 \mathrm{~W})$ & $2.056(2)$ & $\mathrm{Co}(1)-\mathrm{O}(3 \mathrm{~W})$ & $2.135(2)$ \\
$\mathrm{Co}(1)-\mathrm{O}(6 W)$ & $2.094(2)$ & $\mathrm{Co}(1)-\mathrm{O}(5 \mathrm{~W})$ & $2.139(2)$ \\
$\mathrm{O}(1)-\mathrm{P}(1)$ & $1.503(2)$ & $\mathrm{O}(5)-\mathrm{P}(2)$ & $1.497(2)$ \\
$\mathrm{O}(2)-\mathrm{P}(1)$ & $1.576(2)$ & $\mathrm{O}(6)-\mathrm{P}(2)$ & $1.522(2)$ \\
$\mathrm{O}(3)-\mathrm{P}(1)$ & $1.517(2)$ & $\mathrm{P}(1)-\mathrm{C}(3)$ & $1.818(3)$ \\
$\mathrm{O}(4)-\mathrm{P}(2)$ & $1.583(2)$ & $\mathrm{P}(2)-\mathrm{C}(7)$ & $1.808(3)$ \\
\hline
\end{tabular}

3

\begin{tabular}{llll}
\hline $\mathrm{Co}(1)-\mathrm{O}(1 \mathrm{~W})$ & $2.041(4)$ & $\mathrm{Co}(1)-\mathrm{O}(3 \mathrm{~W})$ & $2.081(3)$ \\
$\mathrm{Co}(1)-\mathrm{O}(1 \mathrm{~W}) \# 1$ & $2.041(4)$ & $\mathrm{Co}(1)-\mathrm{O}(2 \mathrm{~W})$ & $2.094(4)$ \\
$\mathrm{Co}(1)-\mathrm{O}(3 \mathrm{~W}) \# 1$ & $2.081(3)$ & $\mathrm{Co}(1)-\mathrm{O}(2 \mathrm{~W}) \# 1$ & $2.094(4)$ \\
$\mathrm{O}(1)-\mathrm{P}(1)$ & $1.531(4)$ & $\mathrm{O}(5)-\mathrm{P}(2)$ & $1.571(4)$ \\
$\mathrm{O}(2)-\mathrm{P}(1)$ & $1.557(4)$ & $\mathrm{O}(6)-\mathrm{P}(2)$ & $1.506(4)$ \\
$\mathrm{O}(3)-\mathrm{P}(1)$ & $1.503(4)$ & $\mathrm{P}(1)-\mathrm{C}(2)$ & $1.789(5)$ \\
$\mathrm{O}(4)-\mathrm{P}(2)$ & $1.529(4)$ & $\mathrm{P}(2)-\mathrm{C}(5)$ & $1.810(5)$ \\
\hline 4 & & & \\
\hline $\mathrm{Ni}(1)-\mathrm{O}(3 \mathrm{~W})$ & $2.010(2)$ & $\mathrm{Ni}(1)-\mathrm{O}(2 \mathrm{~W})$ & $2.070(2)$ \\
$\mathrm{Ni}(1)-\mathrm{O}(6 \mathrm{~W})$ & $2.026(2)$ & $\mathrm{Ni}(1)-\mathrm{O}(5 \mathrm{~W})$ & $2.081(2)$ \\
$\mathrm{Ni}(1)-\mathrm{O}(1 \mathrm{~W})$ & $2.051(2)$ & $\mathrm{Ni}(1)-\mathrm{O}(4 \mathrm{~W})$ & $2.097(2)$ \\
$\mathrm{C}(3)-\mathrm{P}(1)$ & $1.811(3)$ & $\mathrm{O}(3)-\mathrm{P}(1)$ & $1.520(2)$ \\
$\mathrm{C}(7)-\mathrm{P}(2)$ & $1.803(3)$ & $\mathrm{O}(4)-\mathrm{P}(2)$ & $1.585(2)$ \\
$\mathrm{O}(1)-\mathrm{P}(1)$ & $1.499(2)$ & $\mathrm{O}(5)-\mathrm{P}(2)$ & $1.499(2)$ \\
$\mathrm{O}(2)-\mathrm{P}(1)$ & $1.571(2)$ & $\mathrm{O}(6)-\mathrm{P}(2)$ & $1.519(2)$ \\
\hline
\end{tabular}

5

\begin{tabular}{llll}
\hline $\mathrm{Cu}(1)-\mathrm{O}(2)$ & $1.924(3)$ & $\mathrm{Cu}(1)-\mathrm{O}(3) \# 2$ & $1.948(3)$ \\
$\mathrm{Cu}(1)-\mathrm{O}(2) \# 1$ & $1.924(3)$ & $\mathrm{Cu}(1)-\mathrm{O}(3) \# 3$ & $1.948(3)$ \\
$\mathrm{O}(1)-\mathrm{P}(1)$ & $1.577(3)$ & $\mathrm{O}(3)-\mathrm{P}(1)$ & $1.506(3)$ \\
$\mathrm{O}(2)-\mathrm{P}(1)$ & $1.499(3)$ & $\mathrm{P}(1)-\mathrm{C}(1)$ & $1.811(4)$ \\
\hline
\end{tabular}

6

\begin{tabular}{llll}
\hline $\mathrm{Zn}(1)-\mathrm{O}(3 W)$ & $2.062(2)$ & $\mathrm{Zn}(1)-\mathrm{O}(1 \mathrm{~W}) \# 1$ & $2.054(2)$ \\
$\mathrm{Zn}(1)-\mathrm{O}(3 \mathrm{~W}) \# 1$ & $2.062(2)$ & $\mathrm{Zn}(1)-\mathrm{O}(2 \mathrm{~W})$ & $2.100(3)$ \\
$\mathrm{Zn}(1)-\mathrm{O}(1 \mathrm{~W})$ & $2.054(2)$ & $\mathrm{Zn}(1)-\mathrm{O}(2 \mathrm{~W}) \# 1$ & $2.101(3)$ \\
$\mathrm{O}(1)-\mathrm{P}(1)$ & $1.536(2)$ & $\mathrm{O}(5)-\mathrm{P}(2)$ & $1.571(2)$ \\
$\mathrm{O}(2)-\mathrm{P}(1)$ & $1.560(2)$ & $\mathrm{O}(6)-\mathrm{P}(2)$ & $1.501(2)$ \\
$\mathrm{O}(3)-\mathrm{P}(1)$ & $1.503(2)$ & $\mathrm{P}(1)-\mathrm{C}(2)$ & $1.803(3)$ \\
$\mathrm{O}(4)-\mathrm{P}(2)$ & $1.529(2)$ & $\mathrm{P}(2)-\mathrm{C}(5)$ & $1.811(3)$ \\
\hline 7 & & & \\
\hline $\mathrm{Zn}(1)-\mathrm{O}(1)$ & $1.897(2)$ & $\mathrm{Zn}(1)-\mathrm{O}(2 \mathrm{~W})$ & $1.948(3)$ \\
$\mathrm{Zn}(1)-\mathrm{O}(1) \# 1$ & $1.897(2)$ & $\mathrm{Zn}(1)-\mathrm{O}(1 \mathrm{~W})$ & $1.951(3)$ \\
$\mathrm{O}(1)-\mathrm{P}(1)$ & $1.506(2)$ & $\mathrm{O}(3)-\mathrm{P}(1)$ & $1.502(2)$ \\
$\mathrm{O}(2)-\mathrm{P}(1)$ & $1.583(2)$ & $\mathrm{P}(1)-\mathrm{C}(3)$ & $1.800(2)$ \\
\hline 8 & & & $2.323(3)$ \\
\hline $\mathrm{Cd}(01)-\mathrm{O}(3) \# 1$ & $2.248(3)$ & $\mathrm{Cd}(01)-\mathrm{O}(2) \# 3$ & \\
$\mathrm{Cd}(01)-\mathrm{O}(3)$ & $2.248(3)$ & $\mathrm{Cd}(01)-\mathrm{O}(1 \mathrm{~W})$ & $2.326(3)$ \\
$\mathrm{Cd}(01)-\mathrm{O}(2) \# 2$ & $2.323(3)$ & $\mathrm{Cd}(01)-\mathrm{O}(1 \mathrm{~W}) \# 1$ & $2.326(3)$ \\
$\mathrm{O}(1)-\mathrm{P}(1)$ & $1.582(3)$ & $\mathrm{O}(3)-\mathrm{P}(1)$ & $1.509(3)$ \\
$\mathrm{O}(2)-\mathrm{P}(1)$ & $1.512(3)$ & $\mathrm{P}(1)-\mathrm{C}(1)$ & $1.806(4)$
\end{tabular}

${ }^{a}$ Symmetry transformations used to generate equivalent atoms for $\mathbf{1}$ : $\# 1 x,-y, z-1 / 2 ; \# 2-x+1,-y,-z+2 ; \# 3-x+1, y,-z+3 / 2$. For 3 and 6 : $\# 1-x+1,-y+1,-z$. For 5 : $\# 1-x+2,-y+2,-z ; \# 2-x+1,-y+2,-z ; \# 3$ $x+1, y, z$. For 8: $\# 1-x+1, y,-z-1 / 2 ; \# 2-x+1,-y,-z ; \# 3 x,-y, z-1 / 2$. 


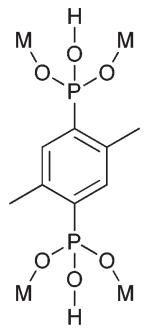

(a)

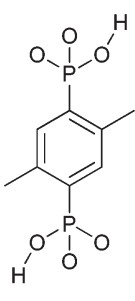

(b)

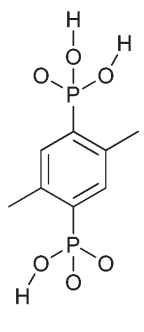

(c)

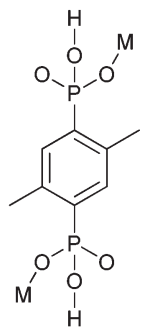

(d)
Scheme 1 Schematic presentation of the coordination modes of the diphosphonate ligands in compounds 1-8.

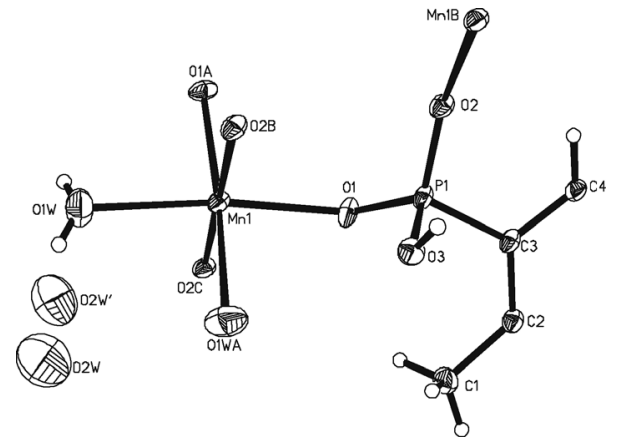

Fig. 1 Coordination environment of the $\mathrm{Mn}(I)$ ion and phosphonate ligand in compound 1 (thermal ellipsoids are given at 30\% probability). Symmetry transformations: A: $1-x, y, 0.5-z ; B: 1-x, 1-y, 1-z ; C: x$, $1-y,-0.5+z$

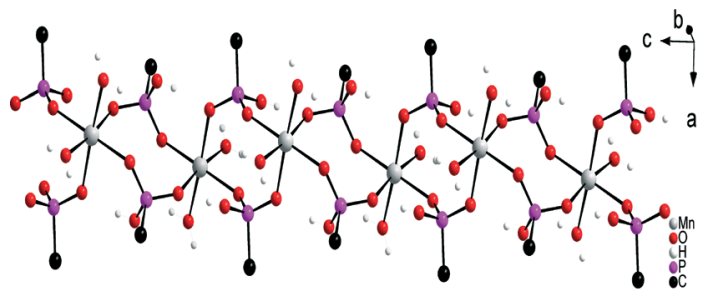

(a)

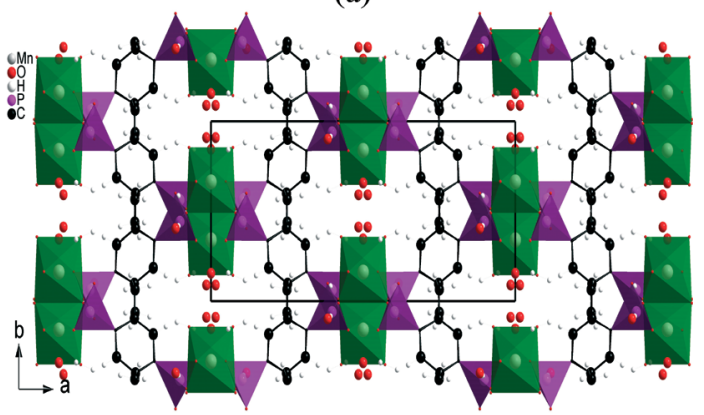

(b)

Fig. 2 One-dimensional infinite chain along the c-direction (a) and three-dimensional packing diagram viewed along the $c$-direction (b) of compound 1. The $\mathrm{CPO}_{3}$ tetrahedrons and $\mathrm{MnO}_{6}$ octahedrons are shaded in pink and green respectively.

mode $b$ in Scheme 1). Both the diphosphonate ligands, the aqua ligands and lattice water molecules can behave

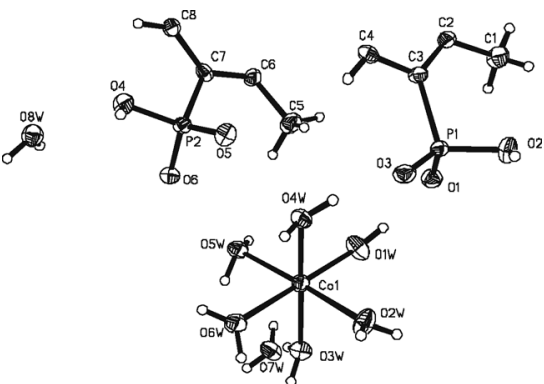

Fig. 3 Coordination environment of the Co(II) ion and phosphonate ligand in compound 2 (thermal ellipsoids are given at $50 \%$ probability).

as donors and acceptors of $\mathrm{O}-\mathrm{H} \cdots \mathrm{O}$ hydrogen bonds (see Table S2, ESI $\dagger)$. It is found that the $\left[\mathrm{Co}\left(\mathrm{H}_{2} \mathrm{O}\right)_{6}\right]^{2+}$ cations are linked into a two-dimensional layer in the $a b$-plane via $\mathrm{O}-\mathrm{H} \cdots \mathrm{O}$ hydrogen bonds with the two lattice water molecules (O7W and O8W, see Fig. 4a). These layers further interact with $\left(\mathrm{H}_{2} \mathrm{~L}\right)^{2-}$ anions through $\mathrm{O}-\mathrm{H} \cdots \mathrm{O}$ interactions and form a three-dimensional supramolecular structure (see Fig. $4 \mathrm{~b}$ and S2, ESI $\dagger$ ).

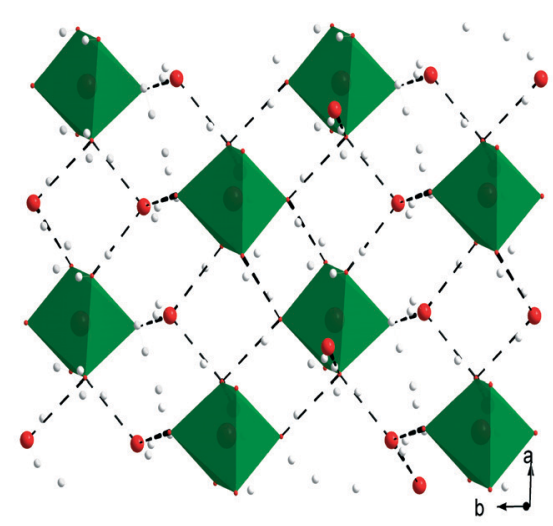

(a)

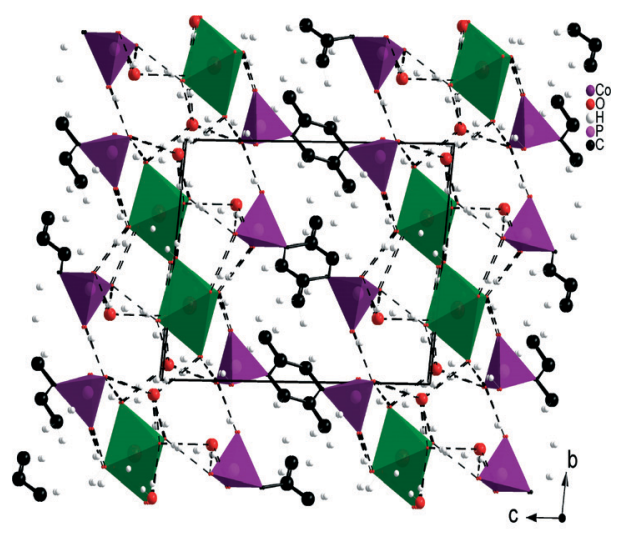

(b)

Fig. 4 Two-dimensional layer in the $a b$-plane (a) and threedimensional packing diagram (b) of compound 2. The $\mathrm{CPO}_{3}$ tetrahedrons and $\mathrm{CoO}_{6}$ octahedrons are shaded in pink and green respectively. $\mathrm{O}-\mathrm{H} \cdots \mathrm{O}$ interactions are shown as dashed lines. 


\section{Structural description of compounds 3 and 6}

When the metal source was changed to Co(II) and Zn(II) chlorides, two isostructural compounds with new structures were obtained. They also exhibit isolated mononuclear structures but have different components compared with those of compounds 2 and 4 . In the asymmetric unit there is one crystallographically independent $\mathrm{Co}$ (II)/Zn(II) ion situated in a special position (0.500000 0.5000000 .000000$)$, one singly deprotonated diphosphonate ligand $\mathrm{H}_{3} \mathrm{~L}^{-}$, three aqua ligands and one lattice water molecule (see Fig. 5 and mode $\mathrm{c}$ in Scheme 1), corresponding to a formula of $\left[\mathrm{M}\left(\mathrm{H}_{2} \mathrm{O}\right)_{6}\right]\left[\left(\mathrm{H}_{3} \mathrm{~L}\right)_{2}\right]\left[\left(\mathrm{H}_{2} \mathrm{O}\right)_{2}\right](\mathrm{M}=\mathrm{Co}$ and $\mathrm{Zn}$ ). The coordinating water molecules (O1w) interact with the lattice water molecules $(\mathrm{O} 4 \mathrm{w})$ strongly, linking the isolated $\left[\mathrm{M}\left(\mathrm{H}_{2} \mathrm{O}\right)_{6}\right]^{2+}(\mathrm{M}=\mathrm{Co}$ and $\mathrm{Zn})$ ions into two-dimensional square grids in the $b c$-plane (see Table S2, ESI $\dagger$ and Fig. 6a). These inorganic layers further interact with the diphosphonate ligands $\mathrm{H}_{3} \mathrm{~L}^{-}$through $\mathrm{O}-\mathrm{H} \cdots \mathrm{O}$ interactions and form a threedimensional supramolecular structure (see Fig. 6b).

\section{Structural description of compound 5}

Compound 5 also crystallizes in the $P \overline{1}$ space group but displays a two-dimensional layered structure. The $\mathrm{Cu}$ (II) center is four-coordinated by four symmetry-related phosphonate oxygen atoms from four diphosphonate ligands, forming a square planar coordination environment (see Fig. 7). The $\mathrm{Cu}-\mathrm{O}$ distances are found in the range of 1.924(3)-1.948(3) $\AA$, which are comparable to those found in other copper phosphonates. ${ }^{17}$ It is worth noting that the coordination environment around the $\mathrm{Cu}$ (II) center can also be regarded as an octahedron if the weak interactions with two symmetryrelated water molecules $(\mathrm{O} 1 \mathrm{w})$ are considered. The $\mathrm{Cu}-\mathrm{O} 1 \mathrm{w}$ distance is $2.846(4) \AA$, obviously longer than the above mentioned four $\mathrm{Cu}-\mathrm{O}$ bonds. The diphosphonate ligand is also doubly deprotonated and exhibits a type a coordination mode (see Scheme 1), which is the same as that found in compound 1. These bridging diphosphonate ligands bridge the $\mathrm{Cu}$ (II) centers into a two-dimensional layered structure (see Fig. 8a). These layers are further assembled into a threedimensional supramolecular structure through static electricity and van der Waals interactions (see Fig. 8b). In 1996, Clearfield and coworkers reported one copper diphosphonate $\mathrm{Cu}_{2}\left[\left(\mathrm{O}_{3} \mathrm{PC}_{6} \mathrm{H}_{4} \mathrm{PO}_{3}\right)\left(\mathrm{H}_{2} \mathrm{O}\right)_{2}\right]^{10 d}$ from 1,4-phenylenediphosphonic acid, but this compound exhibits a three-dimensional lamellar

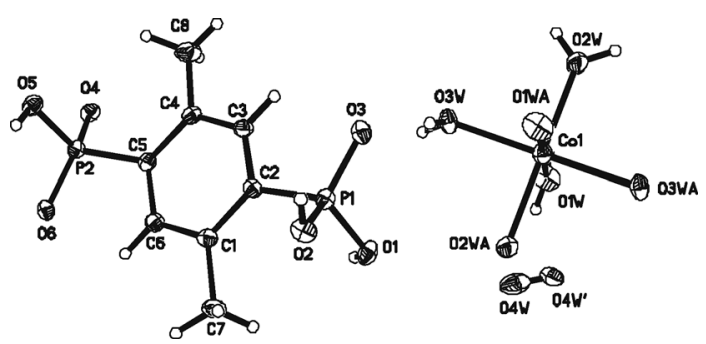

Fig. 5 ORTEP diagram of compound 3 with $30 \%$ probability. Symmetry transformations: A: $1-x, 1-y,-z$.

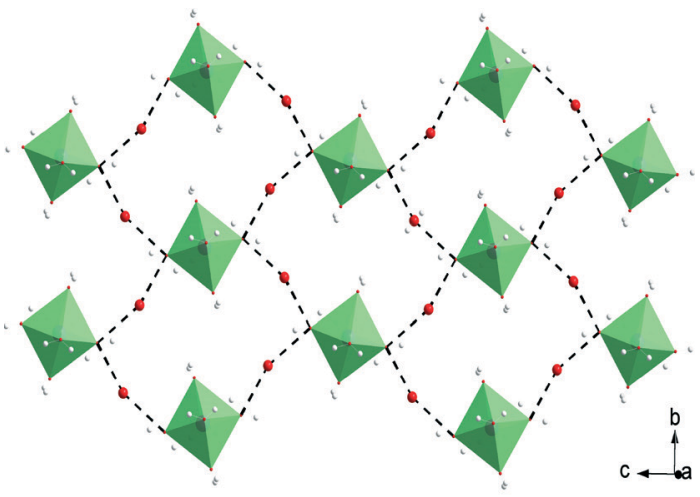

(a)

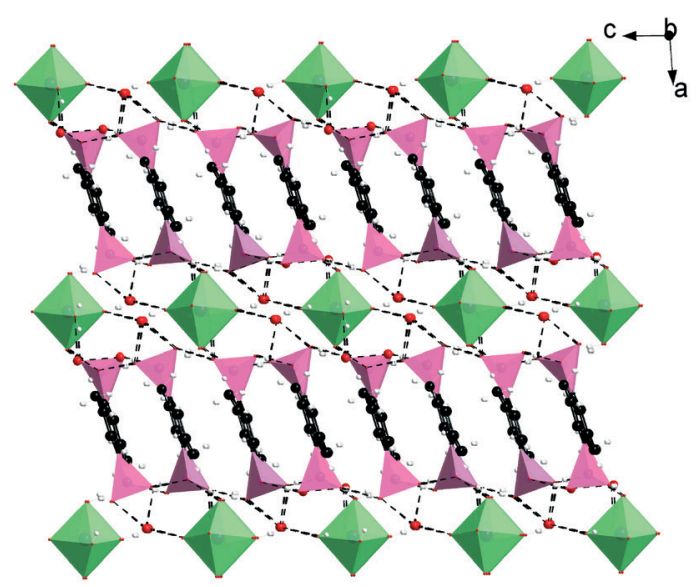

(b)

Fig. 6 Two-dimensional square grids (a) in the bc-plane formed between $\left.\left[\mathrm{M} \mathrm{H}_{2} \mathrm{O}\right)_{6}\right]^{2+}$ cations and $\mathrm{O} 4 \mathrm{~W}$ molecules and threedimensional packing diagram (b) of compounds 3 and 6 , the $-\mathrm{CPO}_{3}$ tetrahedrons and $\mathrm{MO}_{6}(\mathrm{M}=\mathrm{Co}$ and $\mathrm{Zn})$ octahedrons are shaded in pink and green respectively. $\mathrm{O}-\mathrm{H} \cdots \mathrm{O}$ interactions are shown as dark grey dashed lines. O4W' are omitted for clarity.

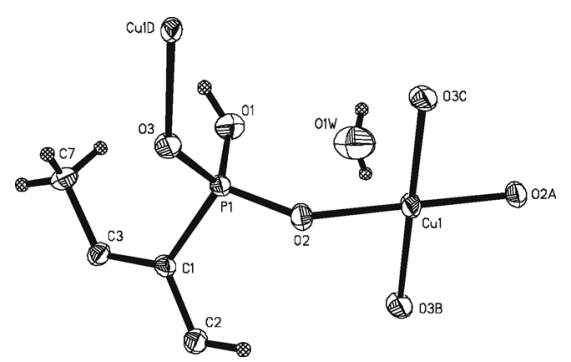

Fig. 7 ORTEP diagram of compound 5 with $50 \%$ probability. Symmetry transformations: A: $2-x, 2-y,-z ; B: 1+x, y, z$; C: $1-x$, $2-y,-z ; \mathrm{D}: x-1, y, z$.

layered structure. This kind of difference stems from the different coordination modes of the $\mathrm{Cu}(\mathrm{II})$ center and the diphosphonate ligand. In $\mathrm{Cu}_{2}\left[\left(\mathrm{O}_{3} \mathrm{PC}_{6} \mathrm{H}_{4} \mathrm{PO}_{3}\right)\left(\mathrm{H}_{2} \mathrm{O}\right)_{2}\right]$, the $\mathrm{Cu}$ (II) center has a distorted square pyramidal coordination geometry and the 1,4-phenylenediphosphonic acid ligand is fully deprotonated, linking with eight $\mathrm{Cu}(\mathrm{II})$ ions. 


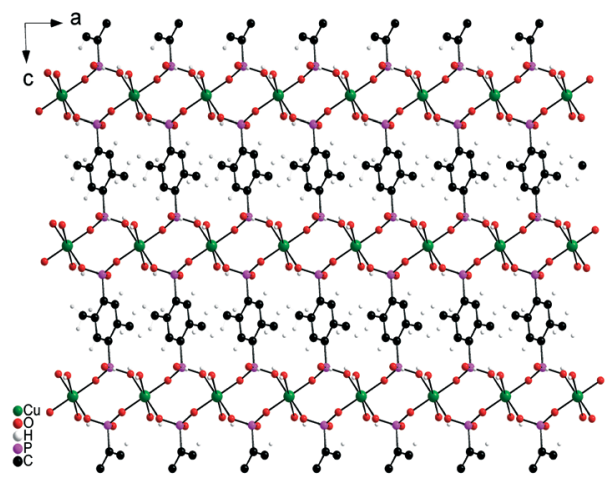

(a)

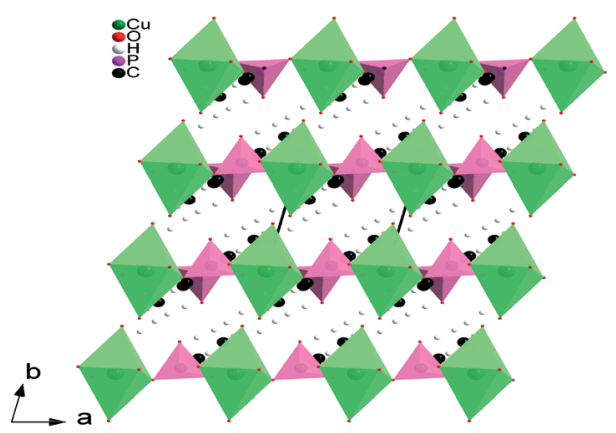

(b)

Fig. 8 Two-dimensional layered structure in the ac-plane (a) and three-dimensional supramolecular structure viewed through the c-direction (b) of compound 5 .

\section{Structural description of compound 7}

Compound 7 crystallizes in the monoclinic space group Pnma with four molecules in each unit cell and possesses a onedimensional chain structure. Each asymmetric unit is comprised of a $\mathrm{Zn}$ (II) ion lying on a mirror plane, half a doubly deprotonated diphosphonate ligand lying about an inversion center and two crystallographically independent aqua ligands lying on a mirror plane, corresponding to a formula of $\left[\mathrm{Zn}\left(\mathrm{H}_{2} \mathrm{~L}\right)\left(\mathrm{H}_{2} \mathrm{O}\right)_{2}\right]$ (see Fig. 9). The $\mathrm{Zn}$ (II) ion is tetrahedrally coordinated by two phosphonate oxygen atoms and two aqua ligands. The $\mathrm{Zn}-\mathrm{O}$ distances fall in the range of 1.897(2)-1.951(3) A, which are also comparable to those of other reported zinc phosphonates. The diphosphonate ligand is also doubly deprotonated but has a bidentate coordination mode (type d, see Scheme 1), which is different to that of 1 and 5 . These bridging bidentate diphosphonate ligands link the $\mathrm{Zn}$ (II) centers into an infinite one-dimensional chain along the $b$-direction (see Fig. 10a). Between the coordinating aqua ligands and uncoordinated phosphonate oxygen atoms, $\mathrm{O}-\mathrm{H} \cdots \mathrm{O}$ interactions are observed (see Table S2, ESI $\dagger$ ), which further assemble the 1D $\mathrm{Zn}$ (II) phosphonate chains into a three-dimensional supramolecular structure (see Fig. 10b). The structure of compound 7 is totally different to that of $\left\{[\mathrm{Zn}(\mathrm{DHBP})](\mathrm{DMF})_{2}\right\}$ $(\mathrm{DHBP}=1,4 \text {-dihydroxy-2,5-benzenediphosphonate })^{10 a}$ and that of $\mathrm{Zn}_{2}\left[\left(\mathrm{O}_{3} \mathrm{PC}_{6} \mathrm{H}_{4} \mathrm{PO}_{3}\right)\left(\mathrm{H}_{2} \mathrm{O}\right)_{2}\right] \cdot{ }^{10 c}$ For $\left\{[\mathrm{Zn}(\mathrm{DHBP})](\mathrm{DMF})_{2}\right\}$, it has a three-dimensional microporous structure in which the $\mathrm{Zn}$ (II)

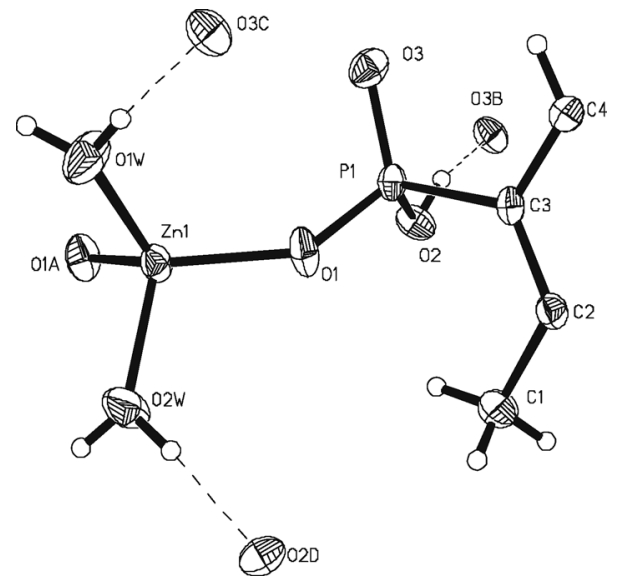

Fig. 9 ORTEP diagram of compound 7 with $50 \%$ probability. Symmetry transformations: A: $x, 1.5-y, z ; B:-0.5+x, y,-0.5-z ; C$ : $0.5+x, y,-0.5-z ; \mathrm{D}: 0.5+x, y, 0.5-z$.

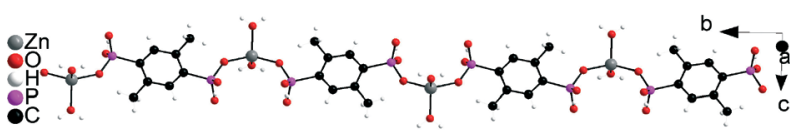

(a)

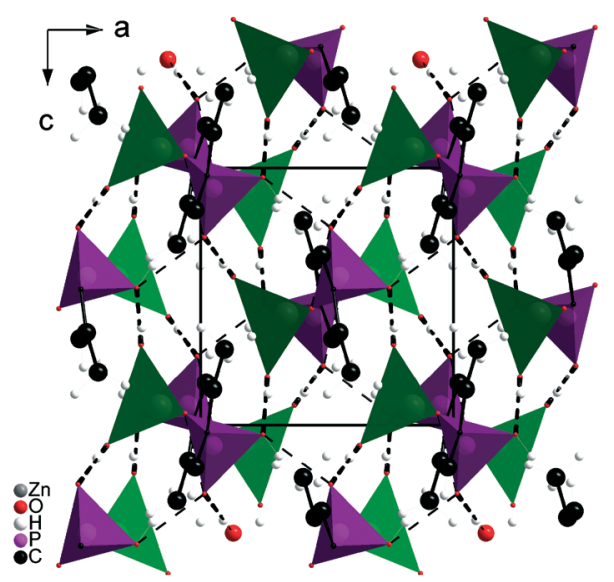

(b)

Fig. 10 One-dimensional infinite chain along the $b$-axis (a) and threedimensional supramolecular structure viewed through the $b$-axis (b) of compound 7. The $\mathrm{O}-\mathrm{H} \cdots \mathrm{O}$ interactions are presented as grey dashed lines.

center is also tetrahedrally coordinated but the DHBP ligand is tetraanionic. $\mathrm{Zn}_{2}\left[\left(\mathrm{O}_{3} \mathrm{PC}_{6} \mathrm{H}_{4} \mathrm{PO}_{3}\right)\left(\mathrm{H}_{2} \mathrm{O}\right)_{2}\right]$ also displays a three-dimensional pillar layered structure but the $\mathrm{Zn}$ (II) center is octahedrally coordinated and the diphosphonate ligand is fully deprotonated.

\section{Structural description of compound 8}

Until now, there has been no report on Cd(II) diphosphonates from 1,4-phenylenediphosphonic acid. The reaction of 2,5-dimethyl-1,4-phenylenediphosphonic acid $\left(\mathrm{H}_{4} \mathrm{~L}\right)$ with $3 \mathrm{CdSO}_{4} \cdot 8 \mathrm{H}_{2} \mathrm{O}$ leads to the formation of compound 8 . The single-crystal X-ray diffraction analysis revealed that the 


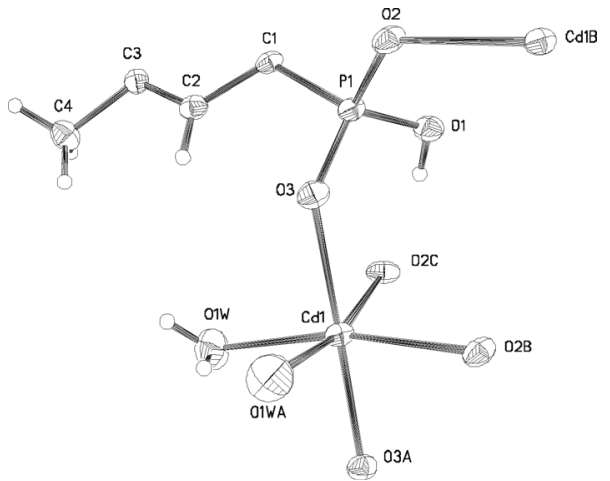

Fig. 11 ORTEP diagram of compound 8 with $50 \%$ probability. Symmetry transformations: A: $1-x, y, 0.5-z$; B: $1-x,-y, 1-z, C: x$, $-y,-0.5+z$.

diphosphonate ligand and the Cd(II) ion have the same coordination modes as those observed for 1 (see Fig. 11), indicating compound 8 is isostructural to 1 (see Fig. S3, ESI $\dagger$ ). However, it is also noted that there is no lattice water molecule in 8 , which is different to that of 1 .

\section{Effect of the ligand decoration on the structure formation}

Among the eight compounds reported in this paper, the corresponding $\mathrm{Co}(\mathrm{II}), \mathrm{Ni}(\mathrm{II}), \mathrm{Cu}(\mathrm{II})$ and $\mathrm{Zn}$ (II) compounds from 1,4-phenylenediphosphonic acid have been reported, but there is no report on the $\mathrm{Cd}$ (II) or $\mathrm{Mn}$ (II) compounds. However, with 2,5-dimethyl-1,4-phenylenediphosphonic acid as the ligand, two three-dimensional $\mathrm{Mn}$ (II) and Cd(II) compounds were successfully obtained, suggesting that the crystallinity of metal phosphonates can be improved by the attachment of methyl groups. It is also noted that the structures of the Co (2 and 3), $\mathrm{Ni}(4), \mathrm{Cu}(5)$ and $\mathrm{Zn}(6)$ compounds reported in this paper are also different from their corresponding analogues synthesized from 1,4-phenylenediphosphonic acid. To make the comparison more convincing, the syntheses were repeated with the same conditions used for the reported metal 1,4-phenylenediphosphonates. ${ }^{10 b-d}$ As expected, the obtained products are either the same as those reported in this paper or are unknown phases (see Table S3 and Fig. S11-S13, ESI†). This kind of difference clearly indicates that the crystal structure of metal diphosphonates based on a rigid phosphonate ligand can be easily altered by attaching methyl groups and provides a new method to fabricate new metal phosphonates with novel structures and interesting properties.

\section{PXRD and thermogravimetric analyses}

Powder X-ray diffraction (PXRD) measurements have been carried out to determine the purities of the obtained bulk samples of compounds 1-8. The good agreement between the simulated and experimental results clearly verify their high purities (see Fig. S4-S10 in the ESI $\dagger$ ).

Thermogravimetric analysis (TGA) measurements were carried out in the temperature range of 25 to $800{ }^{\circ} \mathrm{C}$ to determine the thermal stability of compounds 1 to 8 (see Fig. 12).

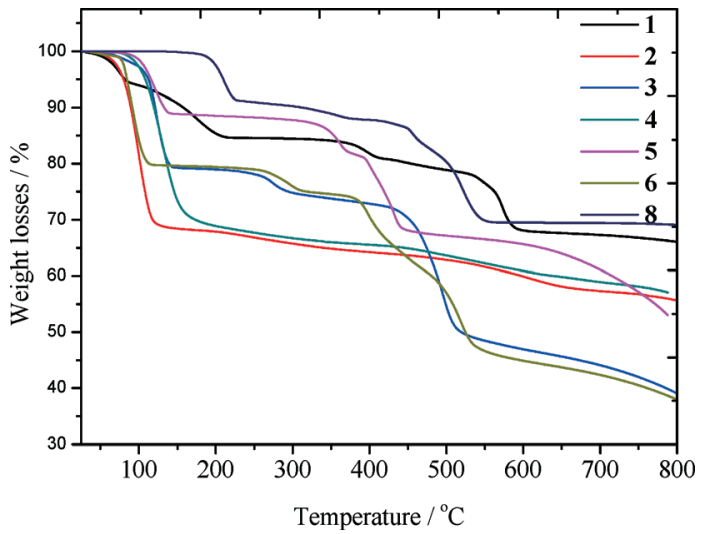

Fig. 12 TGA diagrams of compounds 1-8.

For compound 1, the first three steps of weight losses, occurring in the range of $25-410{ }^{\circ} \mathrm{C}$, can be ascribed to the removal of two lattice and two coordinated water molecules per formula unit. The good agreement between the observed $(18.86 \%)$ and calculated (18.43\%) weight losses clearly confirms this. When the temperature was further elevated to $525{ }^{\circ} \mathrm{C}$, the structure started to collapse. In the case of 2 , the removal of the six aqua ligands and two lattice water molecules happened in the temperature range of $50-126^{\circ} \mathrm{C}$ (calculated: $30.9 \%$; observed: $31.0 \%$ ). The structure of compound 3 is quite similar to that of 2 , but its weight loss behaviour is quite complicated. From the TGA diagram of 3 , it is clear that the weight loss before $147^{\circ} \mathrm{C}$ should be caused by the loss of the six coordinating and two lattice water molecules with a good agreement between the calculated $(19.7 \%)$ and observed $(20.7 \%)$ values. The following two weight losses can be ascribed to the collapse of the structure. For 4, only one abrupt weight loss (30.8\%) can be observed in the range of $75-190{ }^{\circ} \mathrm{C}$, corresponding to the removal of two lattice and six coordinating water molecules $(30.87 \%)$. As for the sample of 5 , the two coordinating water molecules in each formula were removed in the temperature range of $80-140{ }^{\circ} \mathrm{C}$ (observed: $10.99 \%$; calculated: $9.9 \%$ ). After that it was stable until about $320{ }^{\circ} \mathrm{C}$ and then started to decompose upon further heating. As expected, the TGA diagram of 6 is similar to that of 3 , in which the first weight loss $(20.2 \%)$ was observed before $120^{\circ} \mathrm{C}$ (calculated: $19.5 \%$ ). Compound 8 was stable up to $180^{\circ} \mathrm{C}$. After that, the first weight loss occurred and ended at about $230{ }^{\circ} \mathrm{C}$, during which period a weight loss of about $8.76 \%$ was observed, matching well with the calculated value of two coordinating water molecules $(8.74 \%)$. The thermal stability and crystallinity of dehydrated phases of compounds 1, 5 and 8 (obtained by heating the respective as-synthesized samples at 120 and $150{ }^{\circ} \mathrm{C}$ for 6 hours) were also investigated (Fig. S4, S8 and $\mathrm{S} 10, \mathrm{ESI} \dagger)$. The results indicated that all these anhydrous phases were still crystalline. The structures of 5 and 8 were retained but a transformation was observed for 1 , probably due to the strong hydrogen bonds formed between the lattice water molecules and the network. 


\section{Photoluminescence property}

The luminescent properties of $\mathrm{d}^{10}$ metal complexes have been extensively studied for their potential applications in the field of optical materials. Therefore, the solid state luminescent properties of the free ligand $\mathrm{H}_{4} \mathrm{~L}$ as well as compounds 6-8 were investigated at room temperature (see Fig. 13).

Excitation of the powder sample of $\mathrm{H}_{4} \mathrm{~L}$ at $280 \mathrm{~nm}$ at room temperature resulted in a broad emission band with the maximum at $318 \mathrm{~nm}$ and a shoulder at $323 \mathrm{~nm}$, which can be ascribed to the $\pi-\pi^{*}$ transition. Upon excitation at $280 \mathrm{~nm}$, compounds 6-8 also show emissions centered around 319 and $316.5,319.5,324 \mathrm{~nm}$, respectively, with slightly different shapes. These emissions are quite similar to that of the free ligand $\mathrm{H}_{4} \mathrm{~L}$ and therefore should also be ascribed to the intraligand fluorescent emissions. It is also noted that there are two weak peaks at around 422.5 and $454.5 \mathrm{~nm}$ in the emission spectrum of compound 6 , corresponding to either $\pi^{*}-\pi$ or $\pi^{*}-n$ transitions. The different band shapes can be ascribed to their different structures, which can greatly affect the luminescence emission bands, as revealed in the previous literature. ${ }^{18}$

\section{Magnetic property study}

A temperature-dependent magnetic susceptibility measurement for the manganese(II) compound (1) was performed in the temperature range of $2-300 \mathrm{~K}$ at $1 \mathrm{kOe}$ (see Fig. 14).

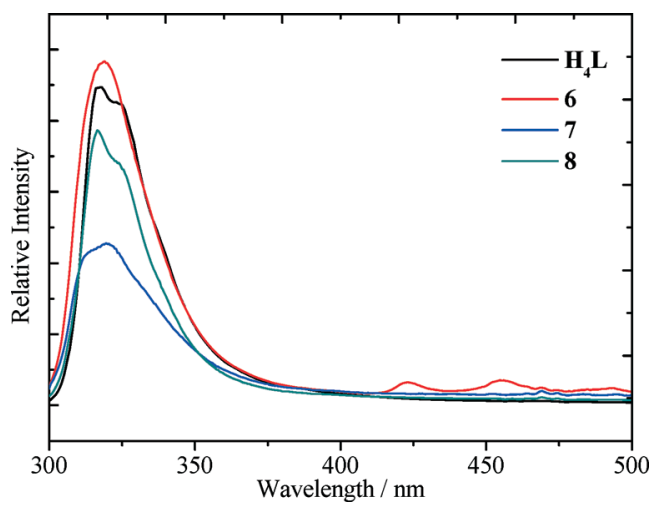

Fig. 13 Emission spectra of ligand $\mathrm{H}_{4} \mathrm{~L}$ and compounds 6-8.

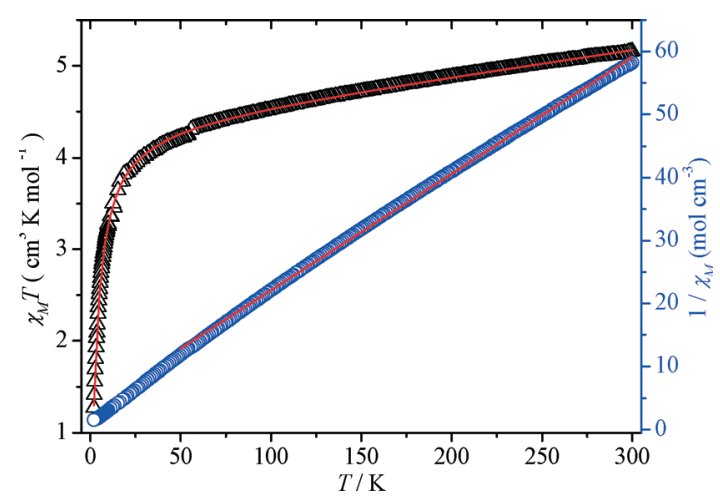

Fig. $14 \chi_{M} T$ and $1 / \chi_{M} T$ versus $T$ plots for compound 1 . Red lines show the best fit of the data according to the proposed model.
Fitting of the susceptibility data according to the Curie-Weiss law gives a Curie constant $(C)$ of 5.4 and a Weiss constant $(\theta)$ of -19.3. At room temperature, the magnetic susceptibility is $5.16 \mathrm{~cm}^{3} \mathrm{~K} \mathrm{~mol}^{-1}$, which is larger than the expected value for one isolated $\mathrm{Mn}^{2+}$ ion with $S=5 / 2$ per formula unit $\left(4.37 \mathrm{~cm}^{3} \mathrm{~K} \mathrm{~mol}^{-1}\right)$. As the temperature is lowered, the $\chi_{\mathrm{M}} T$ value decreases continuously to reach a minimum of $1.28 \mathrm{~cm}^{3} \mathrm{~K} \mathrm{~mol}^{-1}$ at $2 \mathrm{~K}$. Such behavior indicates the presence of overall antiferromagnetic interactions in compound 1 (Fig. 14). The distance between neighboring $\mathrm{Mn}^{2+}$ centers is found to be about $5.376 \AA$, indicating an insignificant interchain interaction or exchange through phosphonate groups ( $\mathrm{Mn}-\mathrm{O}-\mathrm{P}-\mathrm{O}-\mathrm{Mn}$ ) between the $\mathrm{Mn}^{2+}$ centers. It is found that the magnetic exchange interactions can be treated with the $1 \mathrm{D}$ Hamiltonian model $H=-J_{1} \sum S_{2 i} S_{2 i+1}-J_{2} \sum S_{2 i+1} S_{2 i+2},{ }^{19}$ where $J_{1}$ and $J_{2}$ stand for the exchange coupling constants and the $S$ values are classical spin operators. The corresponding analytical expression for the $\chi$ product can be expressed as follows:

$$
\chi_{\text {chain }}=\frac{N g^{2} \mu_{B}^{2}}{3 k T}\left(\frac{1+u_{1}+u_{2}+u_{1} u_{2}}{1-u_{1} u_{2}}\right)
$$

$$
\begin{gathered}
u_{1}=\operatorname{coth}\left[J_{1} S(S+1) /\left(k_{\mathrm{B}} T\right)\right]-\left[k_{\mathrm{B}} T /\left(J_{1} S(S+1)\right)\right] \\
u_{2}=\operatorname{coth}\left[J_{2} S(S+1) /\left(k_{\mathrm{B}} T\right)\right]-\left[k_{\mathrm{B}} T /\left(J_{2} S(S+1)\right)\right] \\
\chi_{\mathrm{M}} T=\frac{\chi_{\text {chain }}}{1-\frac{2 z J \times \chi_{\text {chain }}}{N g^{2} \beta^{2}}}
\end{gathered}
$$

in which $N, g, k$, and $\beta$ have their usual meanings. The best fit gives parameters of $g=2.00, J_{1}=J_{2}=-0.41 \mathrm{~cm}^{-1}$, and $z J=$ -0.14 . These values confirm the antiferromagnetic character of the magnetic interaction between the $\mathrm{Mn}^{2+}$ ions within the 1D chain.

\section{Conclusions}

In summary, a series of metal diphosphonates with variable structures have been synthesized and characterized. The influence of methyl groups on the structure formation of metal phosphonates was investigated. It was found that the attachment of two methyl groups to 1,4-phenylenediphosphonic acid can lead to totally different structures and provides an effective strategy to alter the structures of metal phosphonates. The Zn- and Cd-containing compounds have interesting luminescent properties. The manganese compound shows antiferromagnetic interactions between magnetic centers.

\section{Acknowledgements}

This work was supported by the National Natural Science Foundation of China (no. 21171173 and 21173246). 


\section{Notes and references}

1 K. J. Gagnon, H. P. Perry and A. Clearfield, Chem. Rev., 2012, 112, 1034-1054.

2 M. Eddaoudi, J. Kim, N. Rosi, D. Vodak, J. Wachter, M. O'Keeffe and O. M. Yaghi, Science, 2002, 295, 469-472.

3 (a) M. T. Wharmby, J. P. S. Mowat, S. P. Thompson and P. A. Wright, J. Am. Chem. Soc., 2011, 133, 1266-1269; (b) M. Taddei, F. Costantino, A. Ienco, A. Comotti, P. V. Daud and S. M. Cohen, Chem. Commun., 2013, 49, 1315-1317.

4 J.-G. Mao, Coord. Chem. Rev., 2007, 251, 1493-1520.

5 (a) W. Yang, H. Y. Wu, R. X. Wang, Q. J. Pan, Z. M. Sun and H. Zhang, Inorg. Chem., 2012, 51, 11458-11465; (b) L. Zhang, B. Marzec, R. Clérac, Y. Chen, H. Zhang and W. Schmitt, Chem. Commun., 2013, 49, 66-68; (c) Y. S. Ma, Y. Z. Li, Y. Song and L. M. Zheng, Inorg. Chem., 2008, 47, 4536-4544; (d) P. Ramaswamy, R. Prabhu and S. Natarajan, Inorg. Chem., 2010, 49, 7927-7934.

6 (a) Metal phosphonate chemistry: From synthesis to applications, ed. A. Clearfield and K. D. Demadis, The Royal Society of Chemistry, London, 2012; (b) Z. Chen, Y. Ling, H. Yang, Y. Guo, L. Weng and Y. Zhou, CrystEngComm, 2011, 13, 3378-3382; (c) S. Comby, R. Scopelliti, D. Imbert, L. Charbonnière, R. Ziessel and J.-C. G. Bünzli, Inorg. Chem., 2006, 45, 3158-3160; (d) P. F. Wang, D. K. Cao, S. S. Bao, H. J. Jin, Y. Z. Li, T. W. Wang and L. M. Zheng, Dalton Trans., 2011, 40, 1307-1312; (e) D. K. Cao, M. J. Liu, J. Huang, S. S. Bao and L. M. Zheng, Inorg. Chem., 2011, 50, 2278-2287; $(f)$ H. E. Moll, A. Dolbecq, I. M. Mbomekalle, J. Marrot, P. Deniard, R. Dessapt and P. Mialane, Inorg. Chem., 2012, 51, 2291-2302; (g) Z.-Y. Du, H.-B. Xu and J.-G. Mao, Inorg. Chem., 2006, 45, 6424-6430.

7 S. F. Tang, X. B. Pan, X. X. Lv, S. H. Yan, X. R. Xu, L. J. Li and X. B. Zhao, CrystEngComm, 2013, 15, 1860-1873.

8 S. F. Tang, X. X. Lv, L. J. Li, C. Wang and X. B. Zhao, Inorg. Chem. Commun., 2014, 39, 51-55.

9 (a) Z. Amghouz, J. R. Garcia, S. Garcia-Granda, A. Clearfield, J. Rodriguez Fernandez, I. de Pedro and J. A. Blanco, J. Alloys
Compd., 2012, 536S, S499-S503; (b) V. Zima, J. Svoboda, Y.-C. Yang and S.-L. Wang, CrystEngComm, 2012, 14, 3469-3477; (c) P. O. Adelani and T. E. Albrecht-Schmitt, Cryst. Growth Des., 2011, 11, 4227-4237; (d) Z. Amghouz, S. García-Granda, J. García, A. Clearfield and R. Valiente, Cryst. Growth Des., 2011, 11, 5289-5297; (e) R. Vaidhyanathan, J. Liang, S. S. Iremonger and G. K. H. Shimizu, Supramol. Chem., 2011, 23, 278-282; $(f)$ P. DeBurgomaster, H. Liu, W. Ouellette, C. J. O'Connor and J. Zubiet, Inorg. Chim. Acta, 2010, 363, 4065-4073; (g) A. Subbiah, N. Bhuvanesh and A. Clearfield, J. Solid State Chem., 2005, 178, 1321-1325.

10 (a) J. Liang and G. K. H. Shimizu, Inorg. Chem., 2007, 46, 10449-10451; (b) W. Ouellette, G. Wang, H. Liu, G. T. Yee, C. J. O'Connor and J. Zubieta, Inorg. Chem., 2009, 48, 953-963; (c) D. M. Poojary, B. Zhang, P. Bellinghausen and A. Clearfield, Inorg. Chem., 1996, 35, 5254-5263; (d) D. M. Poojary, B. Zhang, P. Bellinghausen and A. Clearfield, Inorg. Chem., 1996, 35, 4942-4949; (e) D.-K. Cao, S. Gao and L.-M. Zheng, J. Solid State Chem., 2004, 177, 2311-2315.

11 SAINT, Version 6.45, Bruker Analytical X-ray Systems Inc., 2003.

12 G. M. Sheldrick, SADABS, Version 2.10, Bruker AXS Inc., Madison, WI, 2003.

13 G. M. Sheldrick, SHELXS-97, Program for Crystal Structure Solution and Refinement, University of Göttingen, 1997.

14 TWINABS, Bruker AXS, Inc., Madison, WI, 2008.

15 G. M. Sheldrick, SHELXTL, Crystallographic Software Package, SHELXTL, Version 5.1, Bruker-AXS, Madison, WI, 1998.

16 (a) D. Kong and A. Clearfield, Cryst. Growth Des., 2005, 5, 1767-1773; (b) D. Kong, J. Zoñ, J. McBee and A. Clearfield, Inorg. Chem., 2006, 45, 977-986.

17 B. K. Tripuramallu, S. Mukherjee and S. K. Das, Cryst. Growth Des., 2012, 12, 5579-5597.

18 B. Ding, P. Yang, Y. Y. Liu, Y. Wang and G. X. Du, CrystEngComm, 2013, 15, 2490-2503.

19 (a) R. Cortés, M. Drillon, X. Solans, L. Lezama and T. Rojo, Inorg. Chem., 1997, 36, 677-683; (b) F.-Y. Yi, N. Zhao, W. Wu and J.-G. Mao, Inorg. Chem., 2009, 48, 628-637. 